\title{
Glycogen synthase kinase 3 inhibition improves insulin-stimulated glucose metabolism but not hypertension in high-fat-fed C57BL/6J mice
}

\author{
R. Rao • C.-M. Hao • R. Redha • D. H. Wasserman • \\ O. P. McGuinness $•$ M. D. Breyer
}

Received: 14 August 2006 / Accepted: 24 October 2006 / Published online: 7 December 2006

(C) Springer-Verlag 2006

\begin{abstract}
Aims/hypothesis In the current study, the effect of a highly specific peptide inhibitor of glycogen synthase kinase 3 (GSK3) (L803-mts) on glucose metabolism and BP was examined in a high-fat (HF) fed mouse model of diabetes. Methods C57/BL6J mice were placed on an HF diet for 3 months and treated with L803-mts for 20 days, following which glucose metabolism was examined by euglycaemichyperinsulinaemic clamp studies. BP and heart rate were measured by radio-telemetry.

Results The HF mice were obese, with impaired glucose tolerance and high plasma insulin and leptin levels. L803-mts treatment significantly reduced the insulin levels and doubled the glucose infusion rate required to maintain a euglycaemic condition in the HF+L803-mts group compared with the HF group. Insulin failed to suppress the endogenous glucose production rate in the HF group while decreasing it by $75 \%$ in the HF+L803-mts group, accompanied by increased liver glycogen synthase activity and net hepatic glycogen synthesis. GSK3 inhibition also reduced peripheral insulin resistance. Plasma glucose disappearance
\end{abstract}

R. Rao $(\bowtie) \cdot$ C.-M. Hao $\cdot$ R. Redha $\cdot$ M. D. Breyer

S3223, Division of Nephrology, Medical Center North,

Vanderbilt University Medical Center,

Nashville, TN 37232, USA

e-mail: Reena.Rao@Vanderbilt.edu

D. H. Wasserman • O. P. McGuinness • M. D. Breyer

Department of Molecular Physiology and Biophysics,

Vanderbilt University Medical Center,

Nashville, TN, USA

\section{D. Breyer}

Veterans Administration Medical Center,

Vanderbilt University Medical Center,

Nashville, TN, USA rate increased by $60 \%$ in the HF+L803-mts group compared with the HF group. In addition, glucose uptake in heart and gastrocnemius muscle was markedly improved. Although mean arterial pressure increased following the HF diet, it did not change significantly during the 12 days of L803-mts treatment.

Conclusions/interpretation These studies demonstrate that GSK3 inhibition improved hepatic and peripheral insulin resistance in a mouse model of HF-induced diabetes, but it failed to have an effect on BP. GSK3 may represent an important therapeutic target for insulin resistance.

Keywords Euglycaemic-hyperinsulinaemic clamp . Glycogen synthase kinase 3 - High-fat diet · Hypertension . Insulin resistance $\cdot$ L803-mts Peptide inhibitor .

Radio-telemetry

$\begin{array}{ll}\text { Abbreviations } \\ \text { 2-DG } & \text { 2-deoxyglucose } \\ \text { EGP } & \text { endogenous glucose production } \\ \text { G6P } & \text { glucose 6-phosphate } \\ \text { GSK3 } & \text { glycogen synthase kinase } 3 \\ \text { HF } & \text { high fat } \\ \text { HR } & \text { heart rate } \\ \text { MAP } & \text { mean arterial pressure } \\ \text { NF } & \text { normal fat } \\ \text { Rd } & \text { glucose disappearance } \\ \text { Rg } & \text { glucose metabolic index }\end{array}$

\section{Introduction}

Type 2 diabetes mellitus is characterised by peripheral insulin resistance, hyperinsulinaemia and hyperglycaemia. 
The molecular mechanism of insulin resistance involves alterations of multiple signalling pathways in multiple tissues. Glycogen synthase kinase 3 (GSK3) is a constitutively active serine/threonine protein kinase. GSK3 plays an important role in the development of type 2 diabetes, although its precise role remains unclear [1-3]. Studies so far have indicated that GSK3 phosphorylates and inhibits glycogen synthase activity [4,5] and also insulin receptor substrate 1 (IRS-1), thereby impairing insulin signalling.

In diabetes mellitus, GSK3 production and activity levels are elevated [6-8] contributing to the development of insulin resistance [9, 10]. The use of genetically manipulated mice with skeletal-muscle-specific GSK3ß overproduction showed elevated plasma insulin levels and reduced muscle glycogen [10], indicating a specific role of GSK3 in the development of insulin resistance. Insulin promotes conversion of glucose to glycogen by stimulating glucose uptake and activating glycogen synthase. The latter is achieved by activating protein kinase $\mathrm{B}$, which inhibits the GSK $3 \alpha$ or GSK3ß isoforms by phosphorylating their N-terminal residues (Ser21 in GSK3 $\alpha$ and Ser9 in GSK3ß), thus releasing the inhibitory effect of the GSK3 on glycogen synthase $[11,12]$. In mice with constitutively active GSK3ß, insulin failed to activate skeletal muscle glycogen synthase, indicating that insulin normally stimulates skeletal muscle glycogen synthase by reducing GSK3ß activity [3].

Inhibitors of GSK3 have been studied as prospective therapeutic agents for diabetes [9, 13, 14], and several in vitro and in vivo studies have examined their effects on insulin resistance. A few ATP competitive GSK3 inhibitors have been shown to stimulate glycogen synthase activity and improve glucose tolerance in rodent models of diabetes with mutations in leptin $[1,15,16]$ or the leptin receptor [17]. However, the general applicability of these findings and the efficiency of GSK3 inhibitors in other models of insulin resistance has not been demonstrated. In the present study we explored the effect of GSK3 inhibition on glucose metabolism in an environmentally induced (high-fat [HF] diet) insulin-resistant obese mouse model of type 2 diabetes. Several studies have indicated that the C57BL/6J mouse strain on an HF diet is a suitable model for noninsulin-dependent diabetes mellitus and hypertension [1821]. We chose this model because it closely mimics the common form of obesity and insulin resistance associated with human type 2 diabetes. Moreover, this mouse model has been shown to have increased GSK3 activity [7].

Another major concern is the specificity of GSK3 inhibitors. In a recent study, Kaidanovich-Beilin and Eldar-Finkelman [17] investigated the molecular pathway by which GSK3 inhibition by a substrate competitive peptide inhibitor (L803-mts) improves glucose tolerance in $o b / o b$ mice. They observed that IRS-2 and GLUT-4 (also known as GTR4) production levels were higher in liver and muscle, respectively, of L803-mts-treated mice. L803-mts treatment also resulted in decreased phosphoenolpyruvate carboxykinase mRNA levels and higher liver glycogen. In an earlier study, Plotkin et al. [2] had reported that shortterm $(1 \mathrm{~h})$ treatment with the same inhibitor in a diabetic HF-fed C57BL/6J mouse model improved performance on a glucose tolerance test compared with controls. Based on these promising results, we examined if specific inhibition of GSK3 in an insulin-resistant obese C57BL/6J mouse model of type 2 diabetes would improve insulin action and enhance whole-body glucose uptake and metabolism. Studies have shown a direct correlation between insulin resistance and hypertension [22] and stabilisation of plasma insulin levels is associated with a decrease in BP [23]. Hence we also examined the BP and heart rate (HR) of the mice before and after GSK inhibition.

\section{Materials and methods}

Mice Adult (8-week-old) male C57BL/6J mice were purchased from The Jackson Laboratory (Bar Harbor, ME, USA) and housed in a temperature-controlled room on a 12-h light-dark cycle. Mice were placed on either a normal fat (NF) $(n=8)$ or an HF diet $(n=12)$ in which 10 and 58\%, respectively, of total energy were derived from fat (D12329 NF and D12331 HF; Research Diets, New Brunswick, NJ, USA). This HF diet has been shown to induce obesity and mild hypertension in C57BL/6 mice [20]. Body weights were measured weekly. BP measurements and metabolic studies were performed as indicated below. All animal protocols were approved by the Vanderbilt University Institutional Animal Care and Use Committee and followed the 'Principles of laboratory animal care' (NIH publication no. 85-23, revised 1985; http:/www.grants1.nih.gov/grants/ olaw/references/phs).

GSK3 peptide inhibitor A substrate competitive peptide inhibitor of GSK3, L803-mts ( $N$-myristol-GKEAPPAPPQS (p)P) was synthesised by Genemed Synthesis, Inc. (San Francisco, CA, USA) as previously described [2]. Mice were injected i.p. once daily with L803-mts (400 nmol, $300 \mu \mathrm{l}$ ) or vehicle between 10.00 and 11.00 hours for 20 days. BP and HR were determined until the 12th day of treatment, following which the mice underwent surgery for catheter implantation for metabolic studies after the 20th day of L803-mts treatment.

Western blot Frozen gastrocnemius muscles were homogenised and subjected to PAGE. Antibodies used were mouse monoclonal anti-GSK3ß from BD Biosciences (San Jose, CA, USA) and polyclonal anti-phosphylated GSK3 $\alpha, \beta$ and 
anti-phosphylated $\beta$-catenin (Ser33/37/Thr41) from Cell Signaling.

Glucose tolerance test and insulin measurement Glucose tolerance test and insulin measurement Glucose tolerance tests were performed in overnight-fasted $\mathrm{NF}$ and $\mathrm{HF}$ groups. Glucose $(1.5 \mathrm{~g} / \mathrm{kg})$ was injected i.p. and blood samples were collected from a tail vein at various time points. Blood glucose levels were immediately measured with an Accu-Check glucose monitor (Roche Diagnostics, Boehringer Mannheim, Indianapolis, IN, USA). Fasting plasma insulin and leptin were measured by RIA (Linco Research, St Louis, MO, USA).

Serum lipid analysis Serum triacylglycerol was measured using an enzymatic assay adapted to micro-titre plates (Raicham, San Diego, CA, USA).

Euglycaemic-hyperinsulinaemic clamp studies The euglycaemic-hyperinsulinaemic clamp studies were done at the Vanderbilt Mouse Metabolic Phenotyping Center http:// www.mmpc.org) using procedures described by Ayala et al. [24]. Briefly, 3 days before the study, mice were anaesthetised with halothane and an indwelling catheter was inserted into the jugular vein, sealed under the back skin, exteriorised and glued at the back of the neck. Each animal was fasted for $6 \mathrm{~h}$ on the morning of the experiment. Each study consisted of a 2 -h tracer equilibration period ( -120 to $0 \mathrm{~min})$ and a $145-\mathrm{min}$ experimental period $(0-145 \mathrm{~min})$. A primed $(37,000 \mathrm{~Bq} / \mathrm{min})$ continuous $(1,850 \mathrm{~Bq} / \mathrm{min})$ infusion of $\left[3-{ }^{3} \mathrm{H}\right]$ glucose (New England Nuclear, Wilmington, DE, USA) was given through the jugular vein $(-120$ to $0 \mathrm{~min}$ ) to measure glucose turnover. At $t=0 \mathrm{~min}$ the rate of [3- $\left.{ }^{3} \mathrm{H}\right]$ glucose infusion was increased to $3,700 \mathrm{~Bq} / \mathrm{min}$ and insulin was infused at $4 \mathrm{mU} \mathrm{kg}^{-1} \mathrm{~min}^{-1}$ for the duration of the study. Blood glucose levels were measured from $5 \mu \mathrm{l}$ blood every $10 \mathrm{~min}$ using a Hemocue Glucose Analyzer (Angelholm, Sweden). Glucose infusion rate was varied based on on-line blood glucose measurements to clamp blood glucose at $\sim 8.33 \mathrm{mmol} / \mathrm{l}$. Total glucose appearance and glucose disappearance (Rd) were determined by dividing the rate of $\left[3-{ }^{3} \mathrm{H}\right]$ glucose infusion by the plasma $\left[3-{ }^{3} \mathrm{H}\right]$ glucose specific activity. Endogenous glucose production (EGP) was calculated by subtracting the glucose infusion rate from the total glucose appearance. Wholebody glucose clearance was calculated by dividing the mean whole-body $\mathrm{Rd}$ by the mean steady-state plasma glucose concentration obtained during the infusion period. Blood samples were obtained at 0,120 and 145 min for determination of the plasma insulin concentration.

Tissue-specific glucose metabolic index Two hours into the euglycaemic-hyperinsulinaemic clamp studies, a bolus
(481,000 Bq) of ${ }^{14} \mathrm{C}$-labelled 2-deoxyglucose (2-DG) was administered. Blood samples were obtained at 122, 125, $130,135,145 \mathrm{~min}$ to assess plasma 2-DG radioactivity. Mice were anaesthetised after the $t=145 \mathrm{~min}$ blood sample. Vastus lateralis, soleus, gastrocnemius, diaphragm, heart, kidney, adipose tissue and brain were rapidly excised and flash frozen in liquid nitrogen. Tissue-specific glucose metabolic index $(\mathrm{Rg})$ was determined as described earlier $[25,26]$.

Glycogen synthase activity Liver $(75 \mathrm{mg})$ was homogenised in $2.4 \mathrm{ml}$ ice-cold GS homogenisation buffer (35 mmol/l Tris- $\mathrm{HCl} \mathrm{pH} 8.0,8.75 \mathrm{mmol} / \mathrm{l}$ EDTA, $85 \mathrm{mmol} / 1 \mathrm{NaF}, 0.7 \mathrm{~mol} / 1$ sucrose, $0.1 \mathrm{~mol} / 1$ microcystinLR and 'Complete' proteinase inhibitor cocktail). Tissue homogenate was centrifuged and the supernatant fraction snap frozen in liquid nitrogen and stored at $-80^{\circ} \mathrm{C}$. The glycogen synthase activity was measured as described previously [27]. Briefly, $50 \mu \mathrm{l}$ of homogenate were incubated at $30^{\circ} \mathrm{C}$ for $20 \mathrm{~min}$ with $50 \mu \mathrm{l}$ assay buffer containing $10 \%$ glycogen, $10 \mathrm{mmol} / 1$ UDP-glucose (cold), $5,550 \mathrm{~Bq}{ }^{14} \mathrm{C}$-labelled UDP-glucose, $25 \mathrm{mmol} / 1$ sodium sulphate, $50 \mathrm{mmol} / 1 \mathrm{NaF}$ and $27 \mathrm{mmol} / \mathrm{l} \mathrm{EDTA}$, in the presence of 0.1 or $10 \mathrm{mmol} / \mathrm{l}$ glucose 6-phosphate (G6P). From each assay, aliquots of $50 \mu \mathrm{l}$ of the reaction were spotted on 31ETCHR paper and washed three times for $30 \mathrm{~min}$ in $70 \%$ ethanol and once in acetone. After drying, the papers were placed in scintillation vials with $10 \mathrm{ml}$ scintillation liquid and the amount of $\left[{ }^{14} \mathrm{C}\right] U D P$-glucose incorporated into the glycogen was quantified. The glycogen synthase activity ratio is defined as activity measured in the presence of $0.1 \mathrm{mmol} / 1 \mathrm{G} 6 \mathrm{P}$ divided by activity measured in the presence of $10 \mathrm{mmol} / \mathrm{l} \mathrm{G6P}$.

Quantification of liver glycogen Liver (100-200 mg) was homogenised and dissolved by heating in $0.03 \mathrm{~mol} / 1 \mathrm{HCl}$ at $80^{\circ} \mathrm{C}$ for $10 \mathrm{~min}$. Two hundred microlitres of sample were pipetted onto chromatography paper, dried, and washed three times in $66 \%$ alcohol and once with acetone. The paper was cut into strips and incubated with amyloglucosidase in sodium acetate buffer at $37^{\circ} \mathrm{C}$ for $3 \mathrm{~h}$. $\left[{ }^{3} \mathrm{H}\right]$ glucose incorporated into glycogen was determined in digested samples on a Packard Liquid Scintillation Counter. Net hepatic glycogen synthetic rate was calculated by dividing the liver ${ }^{3} \mathrm{H}$ content by plasma $\left[3-{ }^{3} \mathrm{H}\right]$ glucose specific activity during the insulin clamp period. It was assumed that incorporation of radioactive glucose into glycogen was negligible prior to the insulin clamp.

$B P$ measurement by radio-telemetry Initially the BP of six mice each on NF and HF diets was measured to determine if the HF diet induced hypertension in these mice. After establishing a steady baseline, the HF group was treated 
with L803-mts. At the end of BP measurements mice were subjected to metabolic studies. Six HF mice which were sham operated (for transmitter implantation) and vehicleinjected served as controls for the euglycaemic-hyperinsulinaemic clamp studies.

Implantable mouse BP transmitters (TA11PA-C20; Data Sciences International, St Paul, MN, USA) were used to directly measure arterial pressure in individual conscious and freely moving mice [28, 29]. The mice were anaesthetised and the left carotid artery was isolated. The telemetry catheter was inserted into it and the catheter tip advanced into the thoracic aorta such that $3 \mathrm{~mm}$ of the thinwalled tip would reside in the aorta. An s.c. tunnel was formed across the right pectoral area and was enlarged to form a pocket for the transmitter.

All mice were allowed 10 days of recovery to regain their circadian rhythm BP and HR before any measurements were made. Thereafter, BP and HR were telemetrically recorded and stored with the Dataquest ART data acquisition system (Data Sciences International). The baseline measures were averaged over a period of 7 days before the start of the GSK3 inhibitor peptide administration. The BP and HR data were collected over a period of 12 days of inhibitor treatment.

Data analysis Mean arterial pressure (MAP) and HR data collected for 5 and 12 consecutive days before and during GSK3 inhibitor peptide administration, respectively, were plotted as mean values. All data are expressed as means \pm SEM. Statistical analyses of the effects of GSK3 inhibitor were performed with one-way ANOVA to compare changes in BP and HR and two-way ANOVA for repeated measures for metabolic studies (SigmaStat version 3.0; SPSS, Inc., Chicago, IL, USA). Statistical significance was accepted at $p<0.05$.

\section{Results}

Effect of HF diet on body weight and glucose tolerance To establish a mouse model with the characteristic features of type 2 diabetes including obesity, glucose intolerance and hypertension, C57BL/6J mice were placed on an $\mathrm{HF}$ diet for 18 weeks. From the fourth week of feeding, the HF group gained weight rapidly and by the 18 th week it was $144 \%$ of that in the NF group $(50.0 \pm 3.0 \mathrm{~g}$ in HF vs $28.4 \pm$ $2.0 \mathrm{~g}$ in NF, $p<0.005$ ) (Fig. 1a). Fasting plasma insulin levels in the HF group increased by over ninefold $(4.6 \pm 1.0$ vs $0.5 \pm 0.1 \mathrm{ng} / \mathrm{ml}$, HF vs NF, $p<0.001$ ) (Fig. 1a inset) and plasma leptin levels increased by 6.5 -fold compared with NF $(103 \pm 6.2$ vs $15.8 \pm 4.8 \mathrm{pg} / \mathrm{ml}$, HF vs NF, $p<0.001)$. HF feeding for 18 weeks did not render the mice hyper-

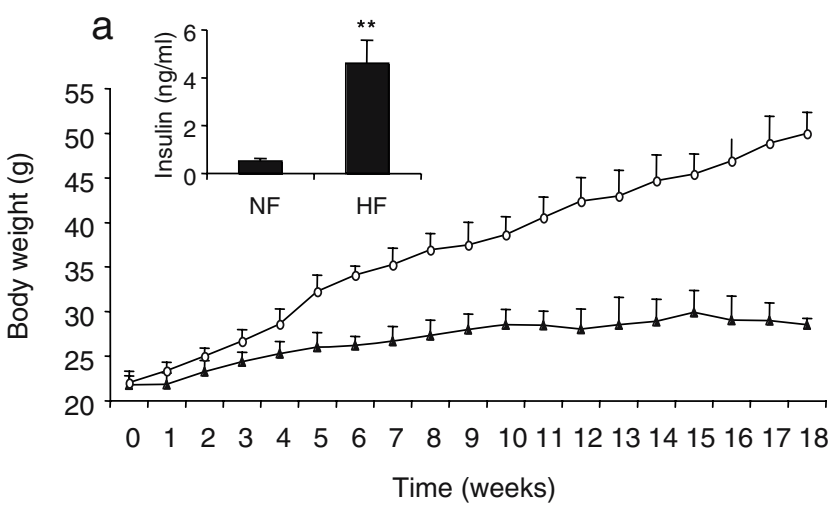

b

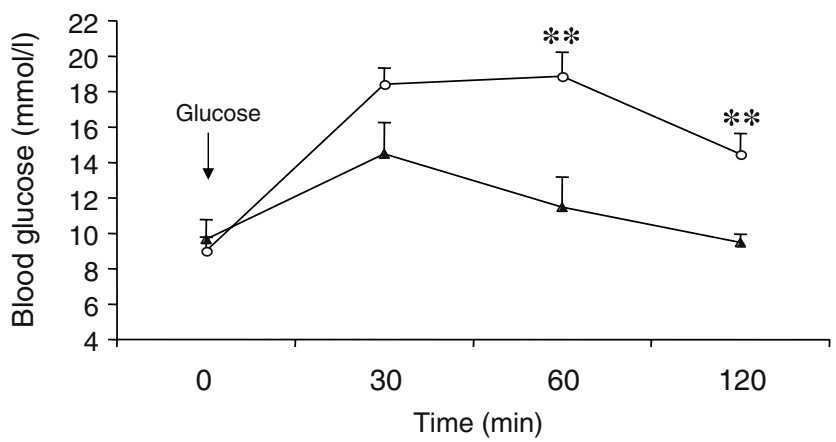

Fig. 1 a Growth rate of C57BL/6J mice fed on NF (filled triangle) or HF (open circles) diet. $n=8$ each group, $p<0.005 \mathrm{NF}$ vs HF. Inset: plasma insulin levels in NF and HF mice, $* * * p<0.001$. b Glucose tolerance test carried out after 18 weeks of HF diet. Mice were starved overnight and blood glucose levels measured before and after i.p. glucose injection $\left(1.5 \mathrm{~g} / \mathrm{kg}\right.$ ). ${ }^{* *} p<0.01$, NF (filled triangles) vs HF (open circles) group, $n=8$

glycaemic, as fasting blood glucose levels were not elevated when compared with the NF group (9.98 \pm $1 \mathrm{mmol} / 1 \mathrm{vs} 9.0 \pm 0.8 \mathrm{mmol} / 1$, NF vs HF group) (Fig. 1b). The HF group showed glucose intolerance in an oral glucose tolerance test, as their blood glucose levels remained high even after $2 \mathrm{~h}$ compared with the NF group (14.5 $\pm 1.2 \mathrm{mmol} / 1$ vs $9.5 \pm 0.5 \mathrm{mmol} / 1 \mathrm{HF}$ vs NF $p<0.01)$.

Effect of GSK3 inhibitor peptide on insulin-stimulated glucose flux To study the effect of GSK3 inhibition on insulin resistance and glucose metabolism, euglycaemichyperinsulinaemic clamp studies were carried out on HFfed mice. The insulin infusion rate $\left(4 \mathrm{mU} \mathrm{kg}^{-1} \mathrm{~min}^{-1}\right)$ was designed to achieve submaximal stimulation of Rd. During the clamp study, the glucose infusion rates in the HF group and the HF+L803-mts groups were adjusted in order to maintain both the groups at euglycaemic conditions. Insulin-stimulated whole-body glucose infusion rate was doubled in the HF+L803-mts group when compared with the HF group (overall, $23 \pm 1$ vs $11 \pm 1.1 \mathrm{mg} \mathrm{kg}^{-1} \mathrm{~min}^{-1}$, $\mathrm{HF}+\mathrm{L} 803$-mts vs HF, $p<0.005$ ) (Fig. 2). This indicates that insulin action is improved by treatment with the GSK3 inhibitor. 


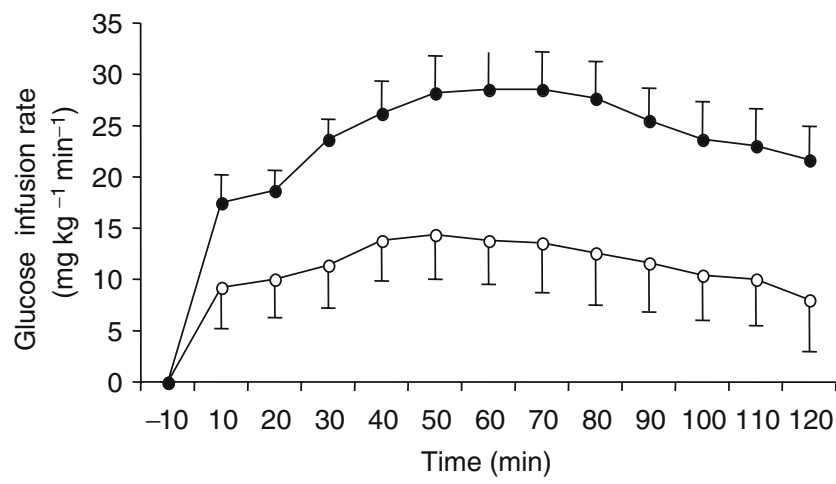

Fig. 2 Glucose infusion rates required to maintain euglycaemic levels during a euglycaemic-hyperinsulinaemic clamp study in HF (open circles) or HF+L803-mts (filled circles) mice, $n=6$ each group, $* p<0.005$ by repeated-measures ANOVA

Insulin failed to suppress EGP in HF mice. In contrast, the EGP in the L803-mts-treated group was suppressed by $75 \%$ (Fig. 3a). Plasma Rd was unchanged during the insulin clamp in the HF group, while in the HF+L803-mts group it increased steadily from $10.6 \pm 1.2$ to $16.5 \pm 1.4 \mathrm{mg} \mathrm{kg}^{-1}$ $\min ^{-1}$ within $80 \mathrm{~min}$ of insulin infusion and remained low
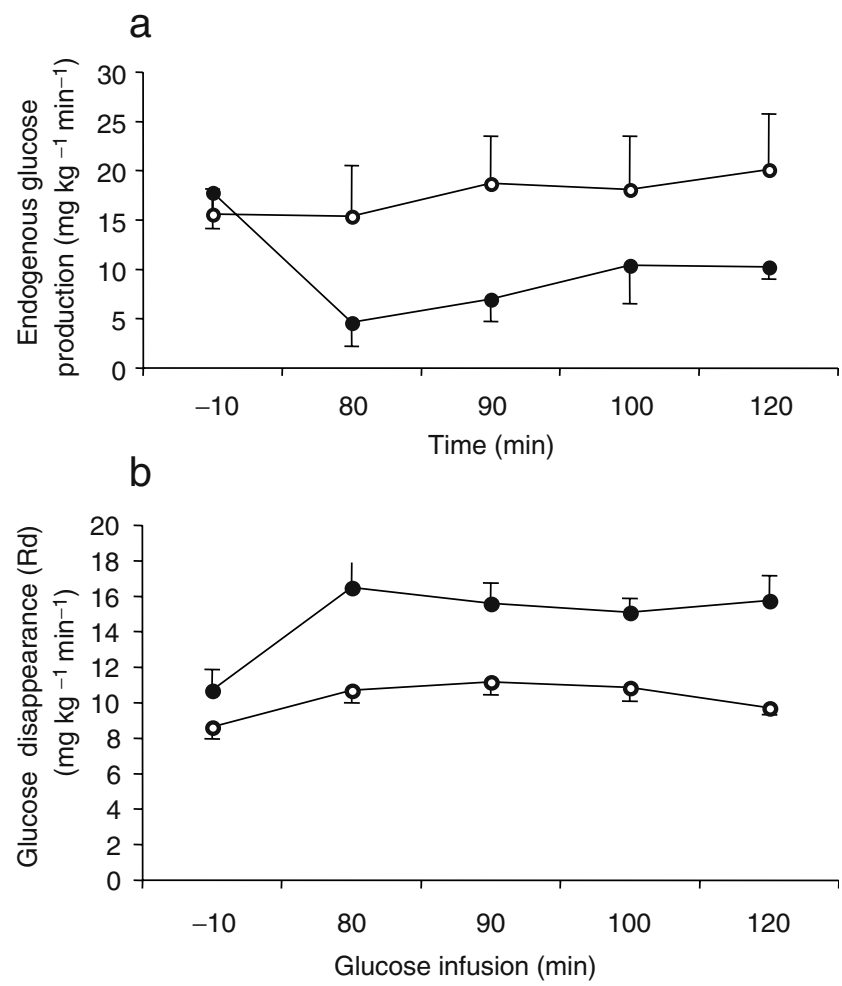

Fig. 3 a Effect of GSK3 inhibition on endogenous glucose production rate calculated by subtracting the glucose infusion rate from the glucose turnover rate; $n=6$ each group, ${ }^{*} p<0.05$, HF (open circles) vs HF+L803-mts (filled circles) mice by repeated-measures ANOVA. b Effect of GSK3 inhibition on whole-body Rd rate was calculated by dividing the mean whole-body glucose turnover rate by the mean steady-state plasma glucose concentration obtained during the infusion period. $n=6,{ }^{*} p<0.05$, HF (open circles) vs HF+L803$\mathrm{mts}$ (filled circles) mice by repeated-measures ANOVA until the end of the study $(p<0.05)$ (Fig. 3b). These results suggest that GSK3 inhibition increases insulin action in HF-fed mice.

Insulin and lipid levels Plasma insulin levels determined before the start of the clamp studies were reduced by $50 \%$ in the HF+L803-mts group compared with the HF group $(1.8 \pm 0.7$ vs $4.0 \pm 1.1 \mathrm{ng} / \mathrm{ml}, p<0.05)$. The insulin levels during the clamp were not different between the two groups. Plasma triacylglycerol and cholesterol levels were also comparable in both groups (triacylglycerol, $1 \pm 0.02 \mathrm{vs}$ $0.98 \pm 0.06 \mathrm{mmol} / \mathrm{l}$, total cholesterol, $4.35 \pm 0.38$ vs $4.6 \pm$ $0.05 \mathrm{mmol} / \mathrm{l}$, HF vs HF+L803-mts).

Effect of GSK3 inhibitor peptide on insulin-stimulated tissue uptake of glucose To examine the effect of GSK3 inhibition on insulin-stimulated tissue uptake of glucose, a bolus of ${ }^{14} \mathrm{C}$-labelled 2-DG was administered to the mice and ${ }^{14} \mathrm{C}$ uptake in various tissues was examined. ${ }^{14} \mathrm{C}$ labelled 2-DG uptake was increased significantly in the heart $\left(0.105 \pm 0.013\right.$ vs $0.252 \pm 0.036 \mu \mathrm{g} \mathrm{min}^{-1} \mathrm{mg}^{-1}$ tissue, $\mathrm{HF}$ vs $\mathrm{HF}+\mathrm{L} 803-\mathrm{mts}, p<0.05)$ and gastrocnemius muscle $\left(0.017 \pm 0.002\right.$ vs $0.041 \pm 0.006 \mu \mathrm{g} \mathrm{min}^{-1} \mathrm{mg}^{-1}$ tissue, HF vs $\mathrm{HF}+\mathrm{L} 803$-mts, $p<0.05$ ). High glucose uptake by muscle indicates that GSK3 inhibition improved peripheral insulin sensitivity (Fig. 4a). To examine if L803-mts treatment and the resulting increase in insulin sensitivity led to changes in muscle GSK3 levels, its protein expression in the gastrocnemius muscle was analysed. Western blot analysis revealed that although the total GSK3ß level was higher in the HF diet group compared with the NF, it did not change in HF+L803-mts group. Similarly, phosphylated GSK $\alpha / \beta$ (Ser9/Ser21), the inactive form of GSK, did not show any change. Since L803-mts is a substrate competitive inhibitor, the level of phosphylated B-catenin (Ser33/ 37/Thr41-phosphorylated by GSK3) was examined as an indicator of GSK activity. Phosphylated B-catenin levels were increased in the HF group compared with the NF group and L803-mts treatment lowered it (Fig. 4b). This indicates that muscle GSK3ß production and activity increased in association with HF-diet-induced insulin resistance, and more importantly, L803-mts treatment lowered GSK activity.

Net liver glycogen synthesis and glycogen synthase activity The effect of L803-mts on rates of net glycogen synthesis was assessed by $\left[3-{ }^{3} \mathrm{H}\right]$ glucose incorporation into liver glycogen. The net glycogen synthesis during the 145-min insulin clamp in the liver of HF mice was sevenfold higher than in HF+L803-mts mice $(0.06 \pm 0.02$ and $0.45 \pm$ $0.13 \mathrm{mg} / \mathrm{g}$, HF vs HF+L803-mts, $p<0.05)$. To determine whether the increased liver glycogen synthesis in L803-mtstreated mice was associated with increased glycogen 


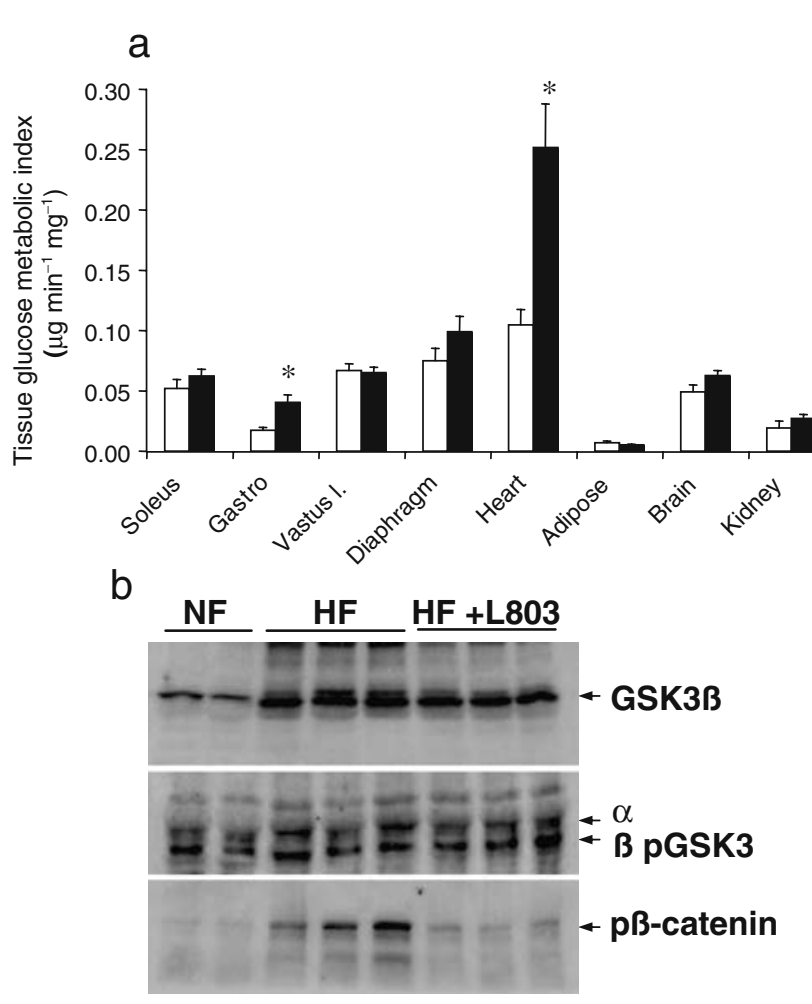

Fig. 4 a Tissue glucose metabolic index. After subjecting the mice to the clamp for $2 \mathrm{~h},{ }^{14} \mathrm{C}$-labelled 2-DG was administered, and after 25 min tissue uptake was measured. HF (open bars) or HF+L803-mts (filled bars) mice, $n=6$ each group, ${ }^{*} p<0.05$. Gastro, gastrocnemius muscle; Vastus $l$, vastus lateralis muscle. b Effect of HF diet or L803-mts treatment on expression of muscle GSK3, phosphylated GSK $(p G S K)$ and phosphylated $\beta$-catenin $(p \beta$-catenin) in NF, HF and $\mathrm{HF}+\mathrm{L} 803$-mts mice

synthase activity, we measured the hepatic glycogen synthase activity by assaying the incorporation of radioactive UDP-glucose into glycogen in the presence or absence of the allosteric activator G6P. In the HF+L803-mts group, glycogen synthase activity ratio was $45 \%$ higher than in the HF group ( $0.20 \pm 0.04$ vs $0.29 \pm 0.02, p<0.05)$ (Fig. 5).

\section{Effect of GSK3 inhibitor peptide on BP and HR To} determine if GSK3 inhibition and improved insulin sensitivity affects BP regulation, HF-fed mice were implanted with radio-telemetric transmitters and GSK3 was systemically inhibited using L803-mts. When compared with the mice on NF diet, the HF group had significantly higher MAP and HR $(109 \pm 3$ vs $117 \pm 1 \mathrm{mmHg}$ and $516 \pm 25$ vs $604 \pm 12$ beats/min; NF vs HF, $p<0.0001$ ) (Fig. 6a,b). Treatment with L803-mts for 12 days did not significantly change MAP. Following GSK3 inhibition, MAP dropped to $115 \pm 1 \mathrm{mmHg}$ by the second day and then gradually increased to $118 \pm 1 \mathrm{mmHg}$ and remained at this level until the end of the study (Fig. 7a). These changes in MAP were not significant. HR decreased significantly from an average $605 \pm 3$ beats per min before treatment to $593 \pm 2$ beats per min after treatment with L803-mts $(p<0.001)$ (Fig. 7b).
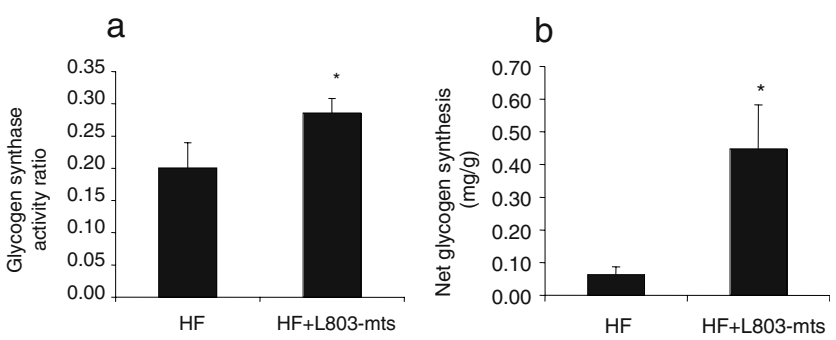

Fig. 5 a Liver glycogen synthase activity expressed as activity measured in the presence of $0.1 \mathrm{mmol} / 1 \mathrm{G} 6 \mathrm{P}$ divided by activity measured in the presence of $10 \mathrm{mmol} / 1 \mathrm{G} 6 \mathrm{P} ; * p<0.05$. b Liver glycogen levels expressed as ${ }^{3} \mathrm{H}$ radioactivity in glycogen. $n=6$ each group, $* p<0.05$

\section{Discussion}

The present studies demonstrate that chronic (20 days) and specific inhibition of GSK3 improves insulin action. GSK3 inhibition did not improve hypertension in HF-fed mice, at least over the short term of this study. Although C57BL/6J mice on the HF diet showed no significant increase in fasting blood glucose, they developed insulin resistance as indicated by impaired glucose tolerance accompanied by high plasma insulin levels compared with mice on an NF diet. Notably, the HF mice had high levels of leptin, which are a closer representation of that seen in individuals with type 2 diabetes than the models lacking leptin or leptin receptors that have been employed in the study of GSK3 inhibitors. When treated with GSK3 inhibitor, circulating plasma insulin levels decreased and glucose tolerance improved. To further confirm the effect of GSK3 inhibition on insulin sensitivity, we used the euglycaemic-hyperinsulinaemic clamp protocol. We found a profound improvement in $\mathrm{Rd}$ rate in the L803-mts-treated mice, associated with an improved suppression of EGP and an augmentation of glucose utilisation, consistent with increased insulin sensitivity.

L803-mts is a synthetic phosphorylated peptide derived from the unconventional recognition motif of GSK3, which can act as its substrate competitive inhibitor. As most protein kinases do not require a pre-phosphorylated site on their substrate as part of their recognition motif, this inhibitor is highly specific for GSK3 [2]. Short-term L803-mts treatment has been shown to mimic insulin action in isolated tissue in vitro by increasing glycogen synthase activity and 2-DG uptake and also improve glucose tolerance in vivo [2]. Here we show using euglycaemic-hyperinsulinaemic clamps that specific inhibition of GSK3 using L803-mts markedly increased wholebody $\mathrm{Rd}$ by improving insulin action in liver and muscle. The improvement in liver insulin action as determined by the clamp was supported by increased net hepatic glycogen synthetic rate and glycogen synthase activity. This is 


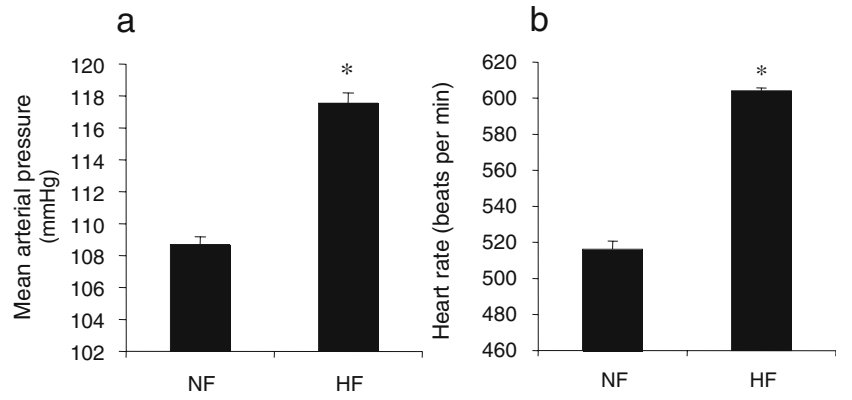

Fig. 6 Mean arterial pressure (a) and HR (b) of NF and HF mice. $n=6$ each group. ${ }^{*} p<0.0001$

consistent with earlier insulin clamp study in Zucker diabetic rats treated with substituted dihydropyrimidines, Chir98014 and Chir98021 [15], which showed that the efficacy of GSK3 inhibition on Rd is mediated primarily by the liver, by increasing glycogen synthase activity and glycogen levels. These studies, however, did not report any change in EGP.

The impaired suppression of EGP contributes to the significantly elevated hyperglycaemia in patients with type 2 diabetes [30]. Studies have indicated that in addition to stimulating glycogen synthesis, selective inhibition of GSK3 could reduce gluconeogenic flux. In the present study, insulin suppressed EGP by $75 \%$ in the L803-mtstreated group, which is consistent with an improvement in hepatic insulin action and the importance of GSK3 in gluconeogenic flux. Insulin controls hepatic glucose production mainly by repression of the gluconeogenic genes phosphoenolpyruvate carboxykinase (Pepck) and G6Pase (G6pc2) [31]. In vitro studies showed GSK3 involvement in the upregulation of $G 6 p c 2$ and Pepck gene expression in the liver [31]. L803-mts treatment reduced Pepck expression by $50 \%$ in $o b / o b$ mice. However, repression of genes involved in gluconeogenesis might not depend entirely on inhibition of GSK3, as shown in non-diabetic double GSK $3 \alpha /$ GSK $3 \beta^{21 \mathrm{~A} / 21 \mathrm{~A}, 9 \mathrm{~A} / 9 \mathrm{~A}}$ knock-in mice, where feeding repressed expression of these genes [32]. It is possible that reversal of insulin resistance by L803-mts treatment in the diabetic mouse model examined in the present study might have made them more responsive to the suppression of EGP by insulin.

Improved insulin sensitivity in L803-mts-treated mice in the present study resulted in part from an improved glucose uptake by heart and gastrocnemius muscle. In the gastrocnemius muscle, GSK3ß production and activity (indicated by phosphylated $\beta$-catenin level) increased in the insulinresistant HF group and the latter was reduced following L803-mts treatment. This is consistent with the observation that L803-mts treatment more than doubled the insulinstimulated $\mathrm{Rg}$ in the gastrocnemius muscle of the HF-fed mice. Although earlier in vitro experiments using a
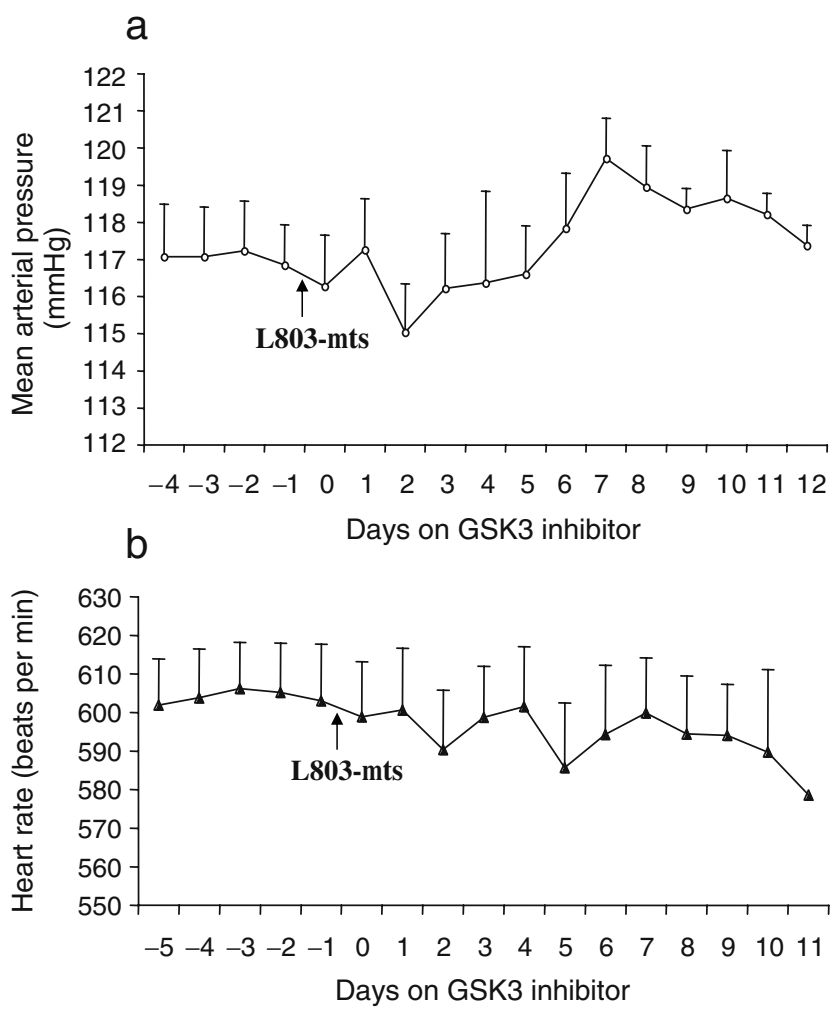

Fig. 7 Mean arterial pressure (a) and HR (b) of HF mice at baseline and after treatment with L803-mts. $n=6$

substituted aminopyrimidine (CT-98014) inhibitor of GSK3 on isolated epitrochlearis and soleus muscle from ZFD rats showed improved insulin-stimulated ${ }^{14} \mathrm{C}$-labelled 2-DG uptake [1, 33], a similar compound, Chir99021, failed to change ${ }^{14} \mathrm{C}$-labelled 2-DG uptake into gastrocnemius muscle in ZDF rats in the only in vivo study reported so far [15]. Similarly, in double GSK $3 \alpha / \mathrm{GSK} 3 \beta^{21 \mathrm{~A} / 21 \mathrm{~A}, 9 \mathrm{~A} / 9 \mathrm{~A}}$ knock-in mice, insulin-stimulated glucose uptake in skeletal muscle was normal, although insulin failed to stimulate glycogen synthase in the gastrocnemius muscle and heart [34]. This is in contrast to the effects of L803-mts on the gastrocnemius and cardiac muscle glucose uptake in the present study.

In the current study, GSK3 inhibition did not improve HF-induced elevation in mean arterial BP in this insulinresistance mouse model. Epidemiological studies indicate that insulin resistance and arterial hypertension are interrelated $[35,36]$. It has been suggested that the prohypertensive action of insulin could be due to increased sodium reabsorption by acting on distinct segments of the renal tubule [37-39]. A feedback mechanism limits insulininduced sodium retention when extracellular fluid volume is expanded [40], which is impaired in rats with hypertension associated with insulin resistance [41]. Inhibition of GSK3 by lithium has been shown to cause urinary concentrating defects [42] and also could inhibit sodium reabsorption in the collecting duct. Renal sodium handling 
is critical for the maintenance of extracellular fluid volume and BP and its impairment has been associated with the development of hypertension [43, 44]. To the best of our knowledge, the possible involvement of renal GSK3 in hypertension has not been examined.

HF diet has been suggested to cause mild hypertension in C57/6J mice [18, 20, 21]. To determine if GSK3 plays a significant role in BP regulation in hyperinsulinaemic and insulin-resistant mice, we treated mice with GSK3 inhibitor peptide. In spite of a significant improvement in insulin sensitivity, these mice did not show a decrease in their MAP, indicating that GSK3 activity in this model of insulin resistance is not linked to hypertension over the course of 12 days. It could be possible that prolonged treatment will be required to see a beneficial effect of GSK3 inhibitor on BP. However, the HR of the mice decreased after treatment with GSK3 inhibitor. This is consistent with our observation that the greatest increase in tissue Rg after L803-mts treatment was in the cardiac muscles. Earlier studies have reported that cardiac muscles have very high levels of GSK3ß [34]. Hence it is possible that GSK3 inhibition has a more direct effect on HR.

In conclusion, long-term GSK3-inhibitor treatment significantly reduced insulin resistance in diabetic mice, as a combined effect of improved glucose uptake and suppression of endogenous glucose production. Improvement in hepatic insulin action was also evident from the increase in net liver glycogen synthetic rate and glycogen synthase activity. GSK3 inhibition did not change the BP in these mice. Since insulin resistance is an important characteristic in patients with type 2 diabetes, these data suggest that GSK3 inhibition represents an important therapeutic target for this disease.

Acknowledgements We thank the Vanderbilt Mouse Metabolic Phenotyping Center (MMPC) for help with the insulin clamp and radio-telemetry studies and T. Santomango for glycogen analysis. These studies were supported by a South East Affiliate Beginner's Grant in Aid from the AHA to R. Rao, DK071876 to C. Hao and DK037097 to M. Breyer, a Vanderbilt Diabetes Training and Research Center Grant DK20593 to A. C. Powers and an MMPC grant DK59637 to D. Wasserman.

Duality of interest The authors declare that they do not have any financial interests that might conflict with this study.

\section{References}

1. Henriksen EJ, Kinnick TR, Teachey MK et al (2003) Modulation of muscle insulin resistance by selective inhibition of GSK-3 in Zucker diabetic fatty rats. Am J Physiol Endocrinol Metab 284: E892-E900

2. Plotkin B, Kaidanovich O, Talior I, Eldar-Finkelman H (2003) Insulin mimetic action of synthetic phosphorylated peptide inhibitors of glycogen synthase kinase-3. J Pharmacol Exp Ther 305:974-980

3. McManus EJ, Sakamoto K, Armit LJ et al (2005) Role that phosphorylation of GSK3 plays in insulin and Wnt signalling defined by knockin analysis. EMBO J 24:1571-1583

4. Eldar-Finkelman H, Argast GM, Foord O, Fischer EH, Krebs E (1996) Expression and characterization of glycogen synthase kinase-3 mutants and their effect on glycogen synthase activity in intact cells. Proc Natl Acad Sci U S A 93:10228-10233

5. Wang Y, Roach PJ (1993) Inactivation of rabbit muscle glycogen synthase by glycogen synthase kinase-3. Dominant role of the phosphorylation of Ser-640 (site-3a). J Biol Chem 268:2387623880

6. Ciaraldi TP, Nikoulina SE, Henry RR (2002) Role of glycogen synthase kinase-3 in skeletal muscle insulin resistance in type 2 diabetes. J Diabetes Its Complicat 16:69-71

7. Eldar-Finkelman H, Schreyer SA, Shinohara MM, LeBoeuf RC, Krebs EG (1999) Increased glycogen synthase kinase-3 activity in diabetes- and obesity-prone C57BL/6J mice. Diabetes 48:16621666

8. Nikoulina SE, Ciaraldi TP, Mudaliar S, Mohideen P, Carter L, Henry RR (2000) Potential role of glycogen synthase kinase-3 in skeletal muscle insulin resistance of type 2 diabetes. Diabetes 49:263-271

9. Woodgett JR (2003) Physiological roles of glycogen synthase kinase-3: potential as a therapeutic target for diabetes and other disorders. Curr Drug Targets Immune Endocr Metabol Disord 3:281-290

10. Pearce NJ, Arch JR, Clapham JC (2004) Development of glucose intolerance in male transgenic mice overexpressing human glycogen synthase kinase-3beta on a muscle-specific promoter. Metabolism 53:1322-1330

11. Shaw M, Cohen P, Alessi D R (1997) Further evidence that the inhibition of glycogen synthase kinase-3beta by IGF-1 is mediated by PDK1/PKB-induced phosphorylation of Ser-9 and not by dephosphorylation of Tyr-216. FEBS Lett 416:307-311

12. Cross DA, Alessi DR, Cohen P, Andjelkovich M, Hemmings BA (1995) Inhibition of glycogen synthase kinase-3 by insulin mediated by protein kinase B. Nature 378:785-789

13. Martinez A, Castro A, Dorronsoro I, Alonso M (2002) Glycogen synthase kinase 3 (GSK-3) inhibitors as new promising drugs for diabetes, neurodegeneration, cancer, and inflammation. Med Res Rev 22:373-384

14. Eldar-Finkelman H (2002) Glycogen synthase kinase 3: an emerging therapeutic target. Trends Mol Med 8:126-132

15. Cline GW, Johnson K, Regittnig W et al (2002) Effects of a novel glycogen synthase kinase-3 inhibitor on insulin-stimulated glucose metabolism in Zucker diabetic fatty (fa/fa) rats. Diabetes 51:2903-2910

16. Dokken BB, Henriksen EJ (2006) Chronic selective glycogen synthase kinase-3 inhibition enhances glucose disposal and muscle insulin action in pre-diabetic obese Zucker rats. Am J Physiol Endocrinol Metab 291:E209-213

17. Kaidanovich-Beilin O, Eldar-Finkelman H (2006) Long-term treatment with novel glycogen synthase kinase-3 inhibitor improves glucose homeostasis in ob/ob mice: molecular characterization in liver and muscle. J Pharmacol Exp Ther 316:17-24

18. Mills E, Kuhn CM, Feinglos MN, Surwit R (1993) Hypertension in $\mathrm{CB} 57 \mathrm{BL} / 6 \mathrm{~J}$ mouse model of non-insulin-dependent diabetes mellitus. Am J Physiol 264:R73-R78

19. Sandu O, Song K, Cai W, Zheng F, Uribarri J, Vlassara H (2005) Insulin resistance and type 2 diabetes in high-fat-fed mice are linked to high glycotoxin intake. Diabetes 54:2314-2319

20. Williams TD, Chambers JB, Roberts LM, Henderson RP, Overton JM (2003) Diet-induced obesity and cardiovascular regulation in C57BL/6J mice. Clin Exp Pharmacol Physiol 30:769-778 
21. Almind K, Kahn CR (2004) Genetic determinants of energy expenditure and insulin resistance in diet-induced obesity in mice. Diabetes 53:3274-3285

22. Ferrannini E, Buzzigoli G, Bonadonna R et al (1987) Insulin resistance in essential hypertension. N Engl J Med 317:350-357

23. Ghazzi MN, Perez JE, Antonucci TK et al (1997) Cardiac and glycemic benefits of troglitazone treatment in NIDDM. The Troglitazone study group. Diabetes 46:433-439

24. Ayala JE, Bracy DP, McGuinness OP, Wasserman DH (2006) Considerations in the design of hyperinsulinemic-euglycemic clamps in the conscious mouse. Diabetes 55:390-397

25. Halseth AE, Bracy DP, Wasserman DH (1999) Overexpression of hexokinase II increases insulin and exercise-stimulated muscle glucose uptake in vivo. Am J Physiol 276:E70-E77

26. Pederson BA, Schroeder JM, Parker GE, Smith MW, DepaoliRoach AA, Roach PJ (2005) Glucose metabolism in mice lacking muscle glycogen synthase. Diabetes 54:3466-3473

27. Thomas JA, Schlender KK, Larner J (1968) A rapid filter paper assay for UDPglucose-glycogen glucosyltransferase, including an improved biosynthesis of UDP-14C-glucose. Anal Biochem 25:486-499

28. Xue B, Pamidimukkala J, Hay M (2005) Sex differences in the development of angiotensin II-induced hypertension in conscious mice. Am J Physiol Heart Circ Physiol 288:H2177-H2184

29. Mills PA, Huetteman DA, Brockway BP et al (2000) A new method for measurement of blood pressure, heart rate, and activity in the mouse by radiotelemetry. J Appl Physiol 88:1537-1544

30. Magnusson I, Rothman DL, Katz LD, Shulman RG, Shulman GI (1992) Increased rate of gluconeogenesis in type II diabetes mellitus. A $13 \mathrm{C}$ nuclear magnetic resonance study. J Clin Invest 90:1323-1327

31. Lochhead PA, Coghlan M, Rice SQ, Sutherland C (2001) Inhibition of GSK-3 selectively reduces glucose-6-phosphatase and phosphatase and phosphoenolpyruvate carboxykinase gene expression. Diabetes 50:937-946

32. Lipina C, Huang X, Finlay D, McManus EJ, Alessi DR, Sutherland C (2005) Analysis of hepatic gene transcription in mice expressing insulin-insensitive GSK3. Biochem J 392: 633-639

33. Ring DB, Johnson KW, Henriksen EJ (2003) Selective glycogen synthase kinase 3 inhibitors potentiate insulin activation of glucose transport and utilization in vitro and in vivo. Diabetes 52:588-595

34. Mora A, Sakamoto K, McManus EJ, Alessi DR (2005) Role of the PDK1-PKB-GSK3 pathway in regulating glycogen synthase and glucose uptake in the heart. FEBS Lett 579:3632-3638

35. Modan M, Halkin H, Almog S et al (1985) Hyperinsulinemia. A link between hypertension obesity and glucose intolerance. J Clin Invest 75:809-817

36. Lucas CP, Estigarribia JA, Darga LL, Reaven GM (1985) Insulin and blood pressure in obesity. Hypertension 7:702-706

37. Kirchner KA (1988) Insulin increases loop segment chloride reabsorption in the euglycemic rat. Am J Physiol 255:F1206F1213

38. DeFronzo RA, Goldberg M, Agus ZS (1976) The effects of glucose and insulin on renal electrolyte transport. J Clin Invest 58:83-90

39. Baum M (1987) Insulin stimulates volume absorption in the rabbit proximal convoluted tubule. J Clin Invest 79:1104-1109

40. Sechi LA, Griffin CA, Schambelan M (1994) Effect of dietary sodium chloride on insulin receptor number and mRNA levels in rat kidney. Am J Physiol 266:F31-F38

41. Sechi LA, Griffin CA, Giacchetti G et al (1996) Abnormalities of insulin receptors in spontaneously hypertensive rats. Hypertension 27:955-961

42. Rao R, Zhang MZ, Zhao et al (2005) Lithium treatment inhibits renal GSK-3 activity and promotes cyclooxygenase 2dependent polyuria. Am J Physiol Renal Physiol 288:F642F649

43. Cowley AW Jr (1997) Role of the renal medulla in volume and arterial pressure regulation. Am J Physiol 273:R1-R15

44. Hall JE, Brands MW, Shek EW (1996) Central role of the kidney and abnormal fluid volume control in hypertension. J Hum Hypertens 10:633-639 